\title{
Awareness and use of peri conceptual folic acid supplements amongst pregnant women at a metropolitan hospital
}

\begin{abstract}
Background: Peri conceptual folic acid prevents $70 \%$ of neural tube defects. Worldwide and in Australia 30-64\% of pregnant women adhere with current peri conceptual folic acid supplementation guidelines and $39-82 \%$ is aware of its timing.
\end{abstract}

Objectives: This paper focuses mostly on awareness of peri conceptual folic acid supplementation in pregnancy.

Design: Prospective cross-sectional interviewer-assisted survey.

Setting: Ipswich Hospital, Ipswich, Queensland, Australia.

Participants: 400 pregnant women, $\leq 20$ week's gestation, attending their "booking in" visit.

Results: Full awareness of the importance of folic acid in pregnancy was evident in $70 \%$ of women and was significantly associated with its adherence $(\mathrm{P}<0.05)$. Full awareness of the peri conceptual timing was evident in $47 \%$ of women and was significantly associated with its adherence $(\mathrm{P}<0.05)$. Full awareness of both the importance and timing of folic acid was evident in $36.5 \%$ of women.

Conclusion: Full awareness of peri conceptual folic acid supplementation in pregnancy is low. More work is needed to address this so that women receive specific information regarding the importance of folic acid as well as its peri conceptual timing.
Volume 9 Issue 6 - 2018

\author{
Kassam Mahomed, Melinda Stanton \\ Women's and Children's Services, Ipswich Hospital and \\ University of Queensland,Australia \\ Correspondence: Prof. Kassam Mahomed, Senior Staff \\ Specialist, Women's and Children's Services, Ipswich Hospital \\ and University of Queensland, Chelmsford Avenue, Ipswich \\ 4305, Australia, Tel +(07)38 I0 I I I , +407034283, \\ Email kassam.mahomad@health.qld.gov.au
}

Received: October 07, 2018| Published: November 08, 2018

Keywords: folic acid, peri conceptual, awareness, pregnancy

\section{Introduction}

Neural tube defects (NTD) result from a failure of the neural tube to close during embryonic development. This begins on day 18 and is complete by day 28 after fertilisation. ${ }^{1,2}$ There is clear evidence that women with a higher intake of folic acid (FA) in the peri-conceptual period (one month before and 3 months after conception) have a decreased risk of NTD. ${ }^{3}$ However, because so many pregnancies are unplanned, women may not initiate folic acid supplementation until some or all of the period of most benefit has already passed. ${ }^{4}$

Adequate use of peri-conceptual folic acid supplementation (PFS) varies considerably within Australia and worldwide ${ }^{5,6}$ and may be influenced by many maternal factors, including age, education, smoking and socioeconomic status. ${ }^{5}$ One strategy to optimise folic acid intake in women of childbearing age involves improving awareness and knowledge concerning the benefits of FA. ${ }^{5}$ Awareness of FA is strongly associated with its use. ${ }^{7,8}$ Awareness of folic acid in prevention of NTD varies from $50-73 \%$ internationally ${ }^{8-10}$ and is around $60 \%$ in Australia. ${ }^{11}$ Within Australia in states such as Queensland where there has been no targeted public health campaign on NTD prevention the figure is likely to be much lower.

There is a dearth of research on FA awareness, awareness of it in NTD prevention and its specific use amongst pregnant women in Queensland.
This study was therefore conducted in an area of Queensland that has a higher socio-economic disadvantaged population compared to the Queensland average. ${ }^{11}$ This paper will only focus on the awareness of folic acid and of its role in NTD prevention amongst pregnant women attending for antenatal care.

\section{Methodology}

This was a prospective cross-sectional survey, over a 7-month period, of women attending for their first booking in visit with the midwife at Ipswich Hospital, Ipswich, Queensland. During the year a total of 1830 women were seen at the antenatal clinic. Women were requested to participate if they spoke good English and were 20 weeks or less pregnant. Clinics were surveyed on each of the five working days of the week with the total of 75 clinic days over the study period. After obtaining informed consent women were interviewed in a private room. A piloted semi-structured interviewerassisted questionnaire was used. Unprompted, open-ended questions were used throughout to minimise response bias and ensure women's awareness was comprehensively assessed.

Data entry and quantitative data analysis were performed using SPSS Statistics Version 19.0. ${ }^{12}$ Bivariate analysis was performed using Pearson's chi-squared test. Logistic regression was performed to generate Odds Ratios, 95\% Confidence Intervals and $P$ values for all socio-demographic characteristics of respondents. Data from open-ended responses were coded and presented as themes. 


\section{Results}

Over a period of 7 months 410 women were eligible; 8 women declined and 2 were excluded as the gestational age was subsequently noted to have been $>20$ weeks by ultrasound scan. Demographics data showed that $49.0 \%$ had high school or less education, $38.8 \%$ were unemployed, $20 \%$ had a total household income of $<\$ 35000$ per annum and $11.8 \%$ had private health insurance. Overweight or obesity was present in $59.8 \%$ and $31 \%$ (124/400) of respondents were still smoking.

Women's awareness of folic acid supplementation (incorporating awareness of importance of folic acid as well as its peri-conceptual timing) was classified as "full awareness" in 36.5\% (146/400; 95\% CI, $31.8-41.2 \%)$ and $87.7 \%(128 / 146)$ of these women were aware of FA prior to pregnancy. "Medium awareness" was present in $24.3 \%$ $(97 / 400)$ and "poor awareness" in 39.3\% (157/400) of respondents. Awareness of PFA was significantly associated with its adherence $(P<0.001)$ with higher awareness increasing adherence (Figure 1). Additionally, awareness of FA prior to pregnancy was significantly associated with use $(P=0.024)$.

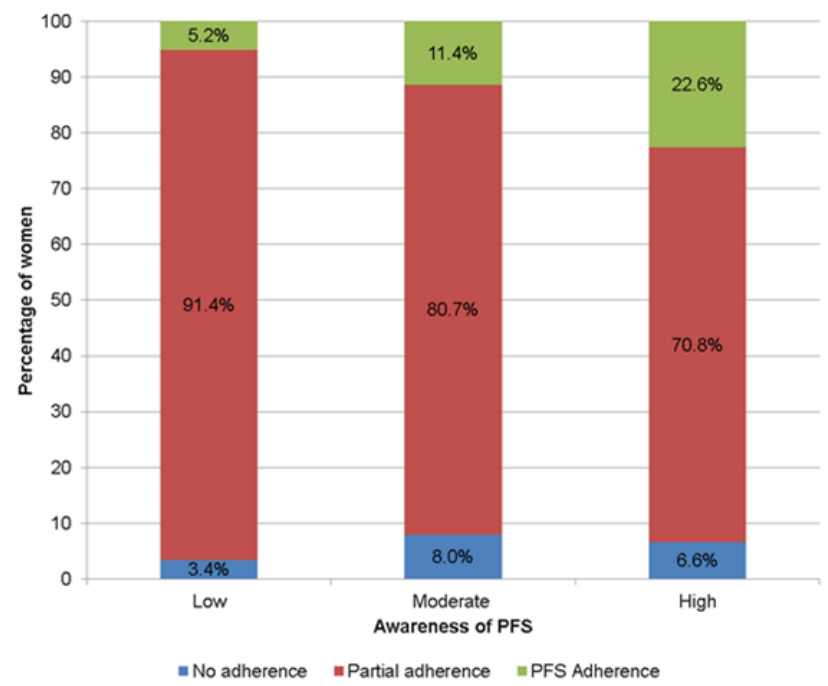

Figure I Relationship between peri conceptual folic acid (PFA) awareness and its adherence.

When asked, "Have you heard of folate or folic acid?" 91.3\% $(365 / 400)$ of women responded positively. Having heard of FA was not significantly related to PFS adherence. Of the women who had heard of FA, $81.1 \%(296 / 365)$ had heard of it prior to pregnancy. Having heard of FA prior to pregnancy was significantly associated with PFS adherence $(P=0.024)$. Women who had not heard of folate or FA were excluded from further analysis of awareness.

\section{Awareness of what substance folic acid}

When asked what substance folate or folic acid the various responses are shown in Table 1. Themes that emerged were categorised as high, moderate and low levels of awareness. A high awareness was present in $32.6 \%(119 / 365)$ of women, who identified it as a B vitamin or vitamin. A moderate level of awareness was present in $15.3 \%(56 / 365)$ of respondents, whilst the largest proportion, $52.0 \%$ $(190 / 365)$, had a low level of awareness. The majority of women with a low level of awareness, $23.8 \%(87 / 365)$, responded that they were unsure what folate or FA was.
Table I Awareness of what folic acid

\begin{tabular}{|c|c|c|}
\hline Substance & $\mathbf{n}$ & $\%$ \\
\hline Total & 365 & 100 \\
\hline High & 119 & 32.6 \\
\hline Vitamin & 117 & 32.1 \\
\hline BVitamin & 2 & 0.5 \\
\hline Moderate & 56 & 15.3 \\
\hline Tablet/ Supplement & 32 & 8.8 \\
\hline Vitamin or Mineral & 23 & 6.3 \\
\hline In green leafy vegetables & 1 & 0.3 \\
\hline Poor & 190 & 52 \\
\hline Unsure & 87 & 23.8 \\
\hline Mineral & 38 & 10.4 \\
\hline Iron & 13 & 3.6 \\
\hline Protein & II & 3 \\
\hline In vegetables & 10 & 2.7 \\
\hline Carbohydrate & 8 & 2.2 \\
\hline Nutrient & 5 & 1.4 \\
\hline Fat & 5 & 1.4 \\
\hline Calcium & 3 & 0.8 \\
\hline Acid & 2 & 0.5 \\
\hline Fruit & 2 & 0.5 \\
\hline In baby formula & 1 & 0.3 \\
\hline Fibre & I & 0.3 \\
\hline By-product of chemical reaction & $\mathrm{I}$ & 0.3 \\
\hline Trace element & $\mathrm{I}$ & 0.3 \\
\hline In fruit and vegetables & $\mathrm{I}$ & 0.3 \\
\hline Pain medication & I & 0.3 \\
\hline
\end{tabular}

\section{Awareness of the importance of folic acid supplements}

When asked if FA supplements are important for women to take $98.4 \%$ (359/365) of women replied affirmatively. These women were asked to explain their reasoning. The themes that emerged from these responses are shown (Table 2). High level awareness of the role of FA in preventing NTD was evident in $70.5 \%(253 / 359)$. High awareness was significantly associated with adequate PFS use $(P=0.002)$. Poor awareness was present in $25.3 \%(91 / 359)$ women - the most common responses were that folic acid was important for growth of the baby $14.5 \%(52 / 359)$ and for pregnancy $7.2 \%(26 / 359)$. The most common incorrect reasons provided for the importance of FA in pregnancy were prevention of Down's syndrome (17 women), cerebral palsy (6 women), cystic fibrosis (2 women) and to help iron levels ( 9 women).

\section{Awareness of the recommended timing of peri- conceptual folic acid supplementation}

In response to the question "When should women take FA supplements?" $47.3 \%$ (189/365) of women's responses were adequate as they encompassed the recommended PFS timing, for at least one 
month prior to conception and for at least the first trimester. Adequate awareness of PFS timing was significantly associated with PFS adherence $(P<0.001)$. Moderate awareness was present in $32.0 \%$ $(117 / 365)$ of women who had nonspecific responses that covered the correct time period. Poor awareness in the remaining $16.2 \%(59 / 365)$ of women encompassed time periods that did not include all of the recommended peri conceptual timing of supplementation (Table 3 ).

Table 2 Awareness of the importance of Folic acid supplements

\begin{tabular}{lll}
\hline Themes & $\mathbf{n}$ & $\%$ \\
\hline Total & 359 & 100 \\
High & 253 & $\mathbf{7 0 . 5}$ \\
Prevents neural tube defects & 21 & 5.8 \\
Prevents spina bifida & 191 & 53.2 \\
Development of/ preventing defects of the baby's & 41 & 11.4 \\
brain/ spinal cord/ back & 15 & 4.2 \\
Moderate & 15 & 4.2 \\
Prevents birth defects & 91 & 25.3 \\
Poor & 52 & 14.5 \\
Growth of baby & 26 & 7.2 \\
pregnancy & 5 & 1.4 \\
Important for women & 3 & 0.8 \\
Important for Iron levels/ prevent anaemia & 1 & 0.3 \\
Was recommended to me - don't know why though & 2 & 0.6 \\
Other & 2 & 0.6 \\
Unsure & &
\end{tabular}

Table 3 Awareness of peri conceptual timing amongst women who have heard of folic acid.

\begin{tabular}{lll}
\hline & $\mathrm{n}$ & $\%$ \\
\hline Total who have heard of folic acid & 365 & 100 \\
\hline Adequate & 189 & 47.3
\end{tabular}

For at least one month prior to conception and for at least the first trimester

\section{Moderate}

Prior to conception and during pregnancy

$\begin{array}{lll}\text { Poor } & \mathbf{5 9} & \mathbf{1 6 . 2} \\ \text { During pregnancy } & 24 & 6.6 \\ \text { During pregnancy and breast feeding } & 10 & 2.7 \\ \text { First trimester } & 4 & 1.1 \\ \text { Unsure } & 12 & 3.3 \\ \text { All the time } & 3 & 0.82 \\ \text { If anaemic } & 1 & 0.27\end{array}$

Awareness of the recommended dose of peri conceptual folic acid supplementation

Recommended dose of PFS was known by only 9.3\% (34/365) of women, whilst $12.3 \%$ (45/365) gave an incorrect dose and $78.4 \%$ (286/365) did not know the recommended dose.

\section{Awareness of folate-rich foods}

Women were prompted to identify which food groups are good sources of folate. The most frequently identified food groups were vegetables $(90.7 \%)$, fruit $(71.5 \%)$, cereals $(65.5 \%)$ and beans/pulses/ legumes/nuts $(64.7 \%)$. The food group though lowest in folate was alcohol (97.5\%) and most respondents were unsure about offal $(47.4 \%)$ as a source of folate.

\section{Awareness of folic acid food fortification}

Nearly $60 \% 218 / 365$ ) of women who had heard of folate were aware of food fortification. The most commonly identified fortified foods were cereals $57.8 \%(126 / 218)$ and bread $41.3 \%$ (90/218).

\section{Socio-demographic and health characteristics related to folic acid awareness}

Socio-demographic factors significantly related to lower FA awareness on univariate analysis were age $<25$ years (OR $2.46,95 \% \mathrm{CI}$ 1.54-3.91, $P<0.001$ ), neither married nor de facto (OR 3.18, 95\%CI 1.60-6.31, $P=0.001$ ), lower education level ( $\leq$ Grade 10 OR 3.41, $95 \% \mathrm{CI} 1.68-6.93, P=0.001$ and lack of private health insurance (OR $2.94,95 \%$ CI $1.37-4.40, \mathrm{P}=0.001)$. In the adjusted model only age $<25$ years $\left(\mathrm{OR}_{\text {adj }} 2.06,95 \% \mathrm{CI} 1.20-3.52, P=0.009\right)$ and lack of private health insurance $\left(\mathrm{OR}_{\mathrm{adj}} 2.36,95 \% \mathrm{CI} 1.13-4.94, \mathrm{P}=0.023\right)$ remained significant.

Health characteristics that were significantly related to lower FA awareness were unplanned pregnancy (OR 2.28, 95\% CI 1.31-3.96, $P=0.004$ ) and not actively avoiding pregnancy (OR 2.01, 95\% CI 1.25-3.22, $P=0.004)$ and pregnancy confirmed at a late gestation (5-8weeks OR 1.91, 95\%CI 1.20-3.04, $P=0.006$; $\geq 9$ weeks OR 4.08, 95\% CI 1.28-12.99, $P=0.017$ ).

\section{Discussion}

This study has shown that nine out of ten women had heard of folic acid, which is amongst the highest reported worldwide ${ }^{8}$. However, having simply heard of FA was not sufficient knowledge; only one in three women were aware that folate or FA is a vitamin, with over half of the women citing an incorrect answer or that they were unsure of what substance FA was.

A more specific awareness of FA during pregnancy was quite low at $70 \%$. Matsuo et al. ${ }^{13}$ also evaluated awareness of FA use among 836 young female college students in Japan. He observed that although $42 \%$ of them had some knowledge of FA, only $9 \%$ had a detailed understanding of the preventive role of FA. This was similar to that reported in South Australia ( $77 \%$ for NTD/spina bifida prevention). ${ }^{14}$ An increasing trend in the percentage of women who are aware of the benefits of folic acid to up to $85 \%$ has been reported from US. ${ }^{15}$

In addition two in every three women had only a moderate or poor level of understanding of the importance and timing of FA recommendations indicating awareness of the peri conceptual use is also still inadequate. This is analogous to the two Australian studies ${ }^{14,16}$ which showed that despite a high level of awareness of the importance of FA, far fewer women (1 in 3) knew the correct peri-conceptual timing.

Socio-demographic characteristics in this study that predict lack of FA awareness were age $<25$ years and lack of private health insurance. Both of these associations have previously been reported 
in Australian ${ }^{14,17}$ and internationally. ${ }^{13,18,19}$ Like us one of these $\mathrm{e}^{20}$ noted that although overall $77.6 \%$ of women surveyed were aware of the benefits of taking FA before pregnancy, this was $32.3 \%$ in the $15-19$ years age group compared to $87 \%$ of those aged $30-34$ and $93 \%$ in the 45-49 years age groups

As we have clearly demonstrated that women of reproductive age or women planning to conceive are mostly unaware of the preventive role of FA, public health promotion initiatives are required. Dissemination needs to occur by government, mass media and medical societies. They also need to be designed to target younger women and women of lower socio-economic grouping, women prior to their childbearing years. ${ }^{19}$ Information strategies for female students as a part of health education or sex education components of school curricula would seem to be quite rational and effective. ${ }^{21}$ They need to be informed about the importance of folic acid as well as the peri conceptual timing of PFS to facilitate the health-related behaviour change. The programs also need to be ongoing, so that each cohort of women is made aware of and takes advantage of the benefits of peri conceptual folic acid supplementation. Too often public health campaigns are only temporary in nature and therefore have no lasting impact. In the Netherlands with over ten years of folic acid health promotion initiatives, the highest rate of its correct use in the world has been demonstrated.?

\section{Acknowledgments}

None.

\section{Conflicts of interest}

The authors have no conflict of interest to declare.

\section{References}

1. Sadler TW. Embryology of neural tube development. Am J Med Genet $C$ Semin Med Genet. 2005;135C(1):2-8.

2. Detrait ER, George TM, Etchevers HC, et al. Human neural tube defects: developmental biology, epidemiology, and genetics. Neurotoxicol Teratol. 2005;27(3):515-524.

3. Lumley J, Watson L, Watson M, et al. Periconceptional supplementation with folate and/or multivitamins for preventing neural tube defects. Cochrane Database Syst Rev. 2001(3):CD001056.

4. Goldberg BB, Alvarado S, Chavez C, et al. Prevalence of Periconceptional Folic Acid Use and Perceived Barriers to the Postgestation Continuance of Supplemental Folic Acid: Survey Results from a Teratogen Information Service. Birth Defects Res A Clin Mol Teratol. 2006;76(3):193-199.

5. Miller EC, Liu N, Wen SW, et al. Why do Canadian women fail to achieve optimal pre-conceptional folic acid supplementation? An observational study. J Obstet Gynaecol Can. 2011;33(11):1116-1123.

6. Abeywardana S, Bower C, Halliday J, et al. Prevalence of neural tube defects in Australia prior to mandatory fortification of bread-making flour with folic acid. Aust N Z J Public Health. 2010;34(4):351-355.
7. de Walle HE, de Jong-van den Berg LT. Ten years after the Dutch public health campaign on folic acid: the continuing challenge. Eur J Clin Pharmacol. 2008;64(5):539-543.

8. CSIRO Health Sciences and Nutrition. E valuating the Folate - Neural Tube Defect Health Claim Pilot. Process Evaluation of the Management Framework. Outcome Evaluation. Part 4 - Appendices, Appendix 4 Consumers' Knowledge about Folate: A report for ANZFA outlining preliminary results from the CSIRO 1998-99 National Dietary Surveys. 2000 .

9. Carmichael SL, Shaw GM, Yang W, et al. Correlates of intake of folic acid-containing supplements among pregnant women. Am J Obstet Gynecol. 2006;194(1):203-210.

10. de Jong-Van den Berg LT, Hernandez-Diaz S, Werler MM, Louik C, et al. Trends and predictors of folic acid awareness and periconceptional use in pregnant women. Am J Obstet Gynecol. 2005;192(1):121-128.

11. Queensland Health. Hospital Activity: Ipswich Hospital, Quarterly hospital activity information for January, February, March 2017. The State of Queensland; 2017.

12. IBM Corporation. IBM SPSS [Computer Program]. 19.0 ed. Somers, New York: IBM Corporation; 2011

13. Matsuo T. Nutrition education on folic acid in college students. Vitamin Japan. 2009;83:277-286.

14. Chan AC, van Essen P, Scott H, et al. Folate awareness and the prevalence of neural tube defects in South Australia, 1966-2007. Med J Aust. 2008;189(10):566-569.

15. Green-Raleigh K, Carter H, Mulinare J, et al. Trends in folic Acid awareness and behavior in the United States: the Gallup Organization for the March of Dimes Foundation surveys, 1995-2005. Matern Child Health J. 2006 Sep;10(5 Suppl):S177-S182.

16. Oddy WH, Miller M, Payne JM, et al. Awareness and consumption of folate-fortified foods by women of childbearing age in Western Australia. Public Health Nutr. 2007;10(10):989-995.

17. Centres for Disease Control and Prevention (CDC). Use of supplements containing folic acid among women of childbearing age--United States, 2007. MMWR Morb Mortal Wkly Rep. 2008;57(1):5-8.

18. Sharp GF, Naylor LA, Cai J, et al. Assessing awareness, knowledge and use of folic acid in Kansas women between the ages of 18 and 44 years. Matern Child Health J. 2009;13(6):814-821.

19. Marsack CR, Alsop CL, Kurinczuk JJ, et al. Pre-pregnancy counselling for the primary prevention of birth defects: rubella vaccination and folate intake. Med J Aust. 1995;162(8):403-406.

20. Nelson CR, Leon JA, Evans J. The relationship between awareness and supplementation: Which Canadian women know about folic acid and how does that translate into use? Can J Public Health. 2014;105(1):e40-e46.

21. Kondo A, Morota N, Date H, et al. Awareness of folic acid use increases its consumption, and reduces the risk of spina bifida. $\mathrm{Br} J$ Nutr. 2015;114(1):84-90. 